\title{
Limit theorems for sums of random variables with mixture distribution
}

\author{
Vladimir Panov \\ International Laboratory of Stochastic Analysis and its Applications \\ National Research University Higher School of Economics \\ Shabolovka, 26, Moscow, 119049 Russia. \\ e-mail: vpanov@hse.ru
}

\begin{abstract}
In this paper, we study the fluctuations of sums of random variables with distribution defined as a mixture of light-tail and truncated heavy-tail distributions. We focus on the case when both the mixing coefficient and the truncation level depend on the number of summands. The aim of this research is to characterize the limiting distributions of the sums due to various relations between these parameters.
\end{abstract}

Keywords and phrases:, mixture distribution, central limit theorem, stable distribution, phase transition.

MSC 2010 subject classifications: Primary 60G50; secondary 60F05, $60 \mathrm{E} 07$.

\section{Introduction}

Theory of limit distributions for the sums of random variables is well-described in brilliant books by Ibragimov and Linnik [6], Meerschaert and Scheffler [7], Petrov [8]. Usually, the most interest is drawn to 2 classical models: a model of i.i.d. random variables and triangular arrays. For the first model, it is common to find non-degenerate laws, which can appear as a limit of the sums $\left(\xi_{1}+\ldots+\right.$ $\left.\xi_{n}\right) b_{n}^{-1}+a_{n}$ with i.i.d. $\xi_{1}, \ldots, \xi_{n}$, and some deterministic sequences $a_{n}, b_{n}$. It is well-known that the set of limiting distributions in this case coincides with the class of stable distributions.

In the second model, one considers an infinitesimal triangular array - a collection of real random variables $\left\{Z_{n k}, k=1 . . k_{n}\right\}, k_{n} \rightarrow \infty$ as $n \rightarrow \infty$, such that $Z_{n 1}, \ldots, Z_{n k_{n}}$ are independent for each $n$ and satisfy the condition of infinite smallness

$$
\sup _{k=1 . . k_{n}} \mathbb{P}\left\{\left|Z_{n k}\right|>\delta\right\} \rightarrow 0, \quad n \rightarrow \infty
$$

for any $\delta>0$. For this model, it is known that only the infinitely divisible distributions can appear as the non-degenerate limit of sums $Z_{n 1}+\ldots+Z_{n k_{n}}-$ $a_{n}$ with deterministic $a_{n}$, and moreover, for any infinitely-divisible distribution there exists a triangular array $\left(Z_{n k}\right)$ such that the sum $Z_{n 1}+\ldots+Z_{n k_{n}}$ converges to this distribution.

Nevertheless, the analysis of the limiting distribution in particular models can be rather tricky. For instance, Ben Arous, Bogachev and Mochanov [1] 
analyzed the asymptotic behaviour of the sums $\sum_{i=1}^{n} e^{t \xi_{i}}$, where $\xi_{1}, \ldots, \xi_{n}$ is an i.i.d. sequence of r.v. with regularly varying log-tail functions, and $n$ and $t$ simultaneously tend to infinity, provided that the speeds of growth of $n$ and $t$ are coordinated via the parameter $\lambda=\liminf _{n, t \rightarrow \infty} \log (n) / \log \left(\mathbb{E}\left[e^{t \xi}\right]\right)$. It turns out, that there exist two critical values of this parameter, $\lambda_{1}<\lambda_{2}$, below which the law of large numbers and the central limit theorem (respectively) break down. This result generalizes some previous findings related to the random energy model, which corresponds to the case when $\xi_{i}$ are standard normal, see Bovier, Kurkova and Löwe [3].

In the current research, we derive similar results for completely another model, defined as a mixture of two distributions: the first distribution has light tails and the second is constructed by truncation of the distribution with heavy tails. More precisely, let $F_{1}(\cdot)$ be a distribution function corresponding to a probability distribution on $\mathbb{R}_{+}$with the upper tail in exponential form, that is,

$$
F_{1}(x)=1-e^{-\lambda x}(1+o(1)), \quad x \rightarrow+\infty,
$$

for some $\lambda>0$. Let $F_{2}(\cdot)$ be a distribution function corresponding to a heavytailed distribution with support on $[1, \infty)$,

$$
F_{2}(x)=1-x^{-\alpha}(1+o(1)), \quad x \rightarrow+\infty,
$$

with $\alpha \in(0,2)$. By $F_{2}^{M}(x)$ denote the truncated distribution function $F_{2}$ at level $M$ :

$$
F_{2}^{M}(x)= \begin{cases}F_{2}(x) / F_{2}(M), & \text { if } x \leq M \\ 0, & \text { if } x>M\end{cases}
$$

Next, consider the mixture of these distributions, that is, the distribution with distribution function

$$
F(x)=(1-\varepsilon) F_{1}(x)+\varepsilon F_{2}^{M}(x),
$$

where $\varepsilon \in(0,1)$ is a mixing parameter, which is assumed to be small. The motivation of considering such mixture goes to the idea to model some "frequent events" by light-tail distributions, and "rare events" by truncated heavy-tailed. For instance, this idea is quite natural for modelling the claim amounts in insurance, see e.g, Rolski et al. [9], or Embrechts, Klüppelberg and Mikosch [4]. Another example comes from population dynamics by analyzing the migration of species. In some models, it is assumed that for most species in the population, the distribution of migration is light-tailed, whereas for some small amount of species the distribution is heavy-tailed, see Whitmeyer and Yang [11].

In this research we consider the case when $\varepsilon$ and $M$ depend on $n$, and moreover, $\varepsilon \rightarrow 0$ and $M \rightarrow \infty$ as $n$ grows. We focus on studying the fluctuations of sums of random variables drawn from the mixture model (2), and aim to characterize the limit laws depending on the relation between $\varepsilon$ and $M$. Several problems of this type are considered in the paper by Grabchak and Molchanov 
[5], where the parameter $M$ as well as both distributions in (2), are fixed. As it is shown in Grabchak and Molchanov [5], the complete asymptotic analysis can be done by taking into account that the distributions are in the domain of attractions of some stable random variables. In this case, it is clear that the limit law for the mixture can be determined by the relation between the normalizing sequences. Nevertheless, this methodology cannot be applied to our set-up, since the parameters $\varepsilon$ and $M$ simultaneously vary.

The paper is organized as follows. In the next section we formulate our main results. It turns out (and is not surprising) that the cases $\alpha \in(0,1)$ and $\alpha \in[1,2)$ are essentially different, see Subsections 2.1 and 2.2 respectively. The proofs are collected in Section 3.

\section{Limit theorems}

Assume that for any $n \in \mathbb{N}$ we are given by $k_{n}=n$ independent random variables $Z_{n 1}, \ldots, Z_{n k_{n}}$ with mixing distribution (2). In other words,

$$
Z_{n k}=\left(1-B_{n k}\right) X_{n k}+B_{n k} Y_{n k}, \quad k=1 . . k_{n},
$$

where $X_{n 1}, \ldots, X_{n k_{n}} \sim F_{1}, Y_{n 1}, \ldots, Y_{n k_{n}} \sim F_{2}^{M_{n}}, B_{n 1}, \ldots, B_{n k_{n}}$ are Bernoulli random variables with probability of success equal to $\varepsilon_{n}$, and all $X_{n k}, Y_{n k}, B_{n k}$, $k=1 . . k_{n}$ are jointly independent for any $n$.

In what follows, we take $M=n^{\gamma_{1}}, \varepsilon=n^{-\gamma_{2}}$ with positive $\gamma_{1}, \gamma_{2}$, and aim to characterize the asymptotic behaviour of the sum $S_{n}:=\sum_{k=1}^{k_{n}} Z_{n k}$ due to the relation between $\gamma_{1}$ and $\gamma_{2}$.

\subsection{Case $\alpha \in(0,1)$}

We start with the most interesting case, $\alpha \in(0,1)$.

Theorem 2.1. $\quad$ (i) Let $\gamma_{1}, \gamma_{2}$ be such that

$$
\gamma_{2}>(2-\alpha) \gamma_{1} \quad \text { or } \quad \gamma_{2}<\min \left\{(2-\alpha) \gamma_{1}, 1-\alpha \gamma_{1}\right\} .
$$

Then the central limit theorem holds, in the sense that

$$
\frac{S_{n}-n \mathbb{E}\left[Z_{n 1}\right]}{\sqrt{n \operatorname{Var}\left(Z_{n 1}\right)}} \stackrel{d}{\longrightarrow} \mathscr{N}(0,1), \quad n \rightarrow \infty
$$

(ii) Let $\gamma_{1}, \gamma_{2}$ be such that

$$
\gamma_{1}>1 / 2 \quad \text { and } \quad \gamma_{2} \in\left(1-\alpha / 2,(2-\alpha) \gamma_{1}\right) .
$$

Then

$$
\frac{S_{n}-n \mathbb{E}\left[X_{n 1}\right]}{\sqrt{n \operatorname{Var}\left(X_{n 1}\right)}} \stackrel{d}{\longrightarrow} \mathscr{N}(0,1), \quad n \rightarrow \infty
$$


(iii) Finally, let $\gamma_{1}, \gamma_{2}$ be such that

$$
\gamma_{1}>1 / 2 \quad \text { and } \quad \gamma_{2} \in\left(\max \left(1-\alpha \gamma_{1}, 0\right), 1-\alpha / 2\right) .
$$

Then for any constant $c>0$

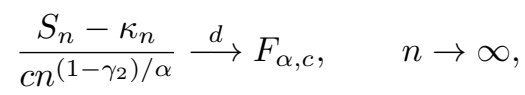

where $F_{\alpha, c}$ is an $\alpha$-stable distribution on $\mathbb{R}_{+}$, that is, an infinitely divisible distribution with the Lévy density $s(x)=c x^{-1-\alpha} I I\{x>0\}$, and without continuous part, and

$$
\kappa_{n}= \begin{cases}n \mathbb{E}\left[X_{n 1}\right], & \text { if } \gamma_{2} \in(1-\alpha, 1-\alpha / 2), \\ n \mathbb{E}\left[X_{n 1}\right]+\frac{c \alpha}{1-\alpha} n^{\left(1-\gamma_{2}\right) / \alpha}, & \text { if } \gamma_{2}=1-\alpha, \\ \frac{c \alpha}{1-\alpha} n^{\left(1-\gamma_{2}\right) / \alpha}, & \text { if } \gamma_{2} \in(0,1-\alpha) .\end{cases}
$$

Note that the normalizing term in (ii) cannot be changed to $\sqrt{n \operatorname{Var}\left(Z_{n 1}\right)}$, and therefore (ii) essentially differs from the central limit theorem. In fact,

$$
\operatorname{Var}\left(Z_{n 1}\right)=\left(\operatorname{Var}\left(X_{n 1}\right)+\frac{2 \alpha}{2-\alpha} n^{(2-\alpha) \gamma_{1}-\gamma_{2}}\right)(1+o(1)), \quad n \rightarrow \infty
$$

and hence $\operatorname{Var}\left(Z_{n 1}\right) \asymp \operatorname{Var}\left(X_{n 1}\right)$ if and only if $\gamma_{2}>(2-\alpha) \gamma_{1}$.

Theorem 2.2. (i) Let $\gamma_{1}, \gamma_{2}$ be such that

$$
\gamma_{2}>(1-\alpha) \gamma_{1} \quad \text { or } \quad \gamma_{2}<\min \left\{(1-\alpha) \gamma_{1}, 1-\alpha \gamma_{1}\right\} .
$$

Then the law of large numbers holds, in the sense that

$$
\frac{S_{n}}{n \mathbb{E}\left[Z_{n 1}\right]} \stackrel{p}{\longrightarrow} 1, \quad n \rightarrow \infty .
$$

(ii) Let $\gamma_{1}, \gamma_{2}$ be such that

$$
\gamma_{2}>1-\alpha \quad \text { and } \quad \gamma_{2} \in\left(1-\alpha,(1-\alpha) \gamma_{1}\right)
$$

Then the analogue of the law of large numbers with normalization $n \mathbb{E}\left[X_{n 1}\right]$ holds, i.e.,

$$
\frac{S_{n}}{n \mathbb{E}\left[X_{n 1}\right]} \stackrel{p}{\rightarrow} 1, \quad n \rightarrow \infty .
$$

Analogously to (6), we note that

$$
\mathbb{E}\left[Z_{n 1}\right]=\left(\mathbb{E}\left[X_{n 1}\right]+\frac{\alpha}{1-\alpha} n^{(1-\alpha) \gamma_{1}-\gamma_{2}}\right)(1+o(1)), \quad n \rightarrow \infty,
$$

and therefore $\mathbb{E}\left[Z_{n 1}\right] \asymp \mathbb{E}\left[X_{n 1}\right]$ if and only if $\gamma_{2}>(1-\alpha) \gamma_{1}$.

Figure 1 illustrates the division of the area $\left(\gamma_{1}, \gamma_{2}\right) \in \mathbb{R}_{+} \times \mathbb{R}_{+}$into subareas with different asymptotic properties of the sums $\sum_{k=1}^{n} X_{n k}$. 


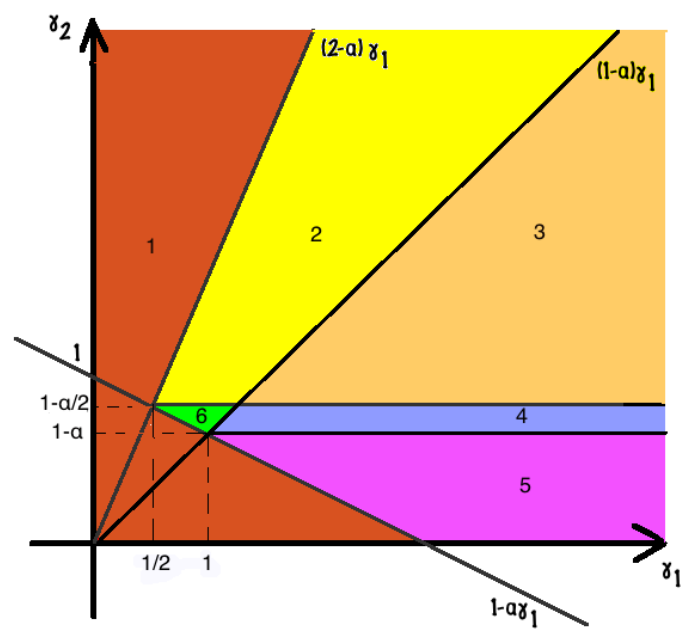

FIG 1. Illustration of the limit behavior of the sums $\sum_{k=1}^{n} X_{n k}$ depending on $\gamma_{1}$ and $\gamma_{2}$ for the case $\alpha \in(0,1)$.

zone 1(red): both the central limit theorem and the law of large numbers hold (see Theorem 2.1(i) and Theorem 2.2(i));

zone 2 (yellow): convergence to the standard normal distribution under normalization $n \mathbb{E}\left[X_{n 1}\right]$ and the law of large numbers (see Theorem 2.1(ii) and Theorem 2.2(i));

zone 3 (orange): convergence to the standard normal distribution under normalization $n \mathbb{E}\left[X_{n 1}\right]$ and the analogue of the LLN with the same normalization (see Theorem 2.1(ii) and Theorem 2.2(ii));

zone 4 (blue): convergence to stable distribution and the analogue of the LLN with normalization $n \mathbb{E}\left[X_{n 1}\right]$ (see Theorem 2.1(iii) and Theorem 2.2(ii));

zone 5 (purple): convergence to stable distribution (see Theorem 2.1(iii));

zone 6 (green): convergence to stable distribution and LLN (see Theorem 2.1(iii) and Theorem 2.2(i)). 


\subsection{Case $\alpha \in[1,2)$}

In this case, the limit laws are more simple. We formulate the results in the next theorem.

Theorem 2.3. Let $\alpha \in[1,2)$. Then

(i) for any positive $\gamma_{1}, \gamma_{2}$ the law of large numbers holds, i.e.,

$$
\frac{S_{n}}{n \mathbb{E}\left[Z_{n 1}\right]} \stackrel{p}{\longrightarrow} 1, \quad n \rightarrow \infty ;
$$

(ii) if $\gamma_{1}, \gamma_{2}$ are such that

$$
\gamma_{2}>(2-\alpha) \gamma_{1} \quad \text { or } \quad \gamma_{2}<\min \left\{(2-\alpha) \gamma_{1}, 1-\alpha \gamma_{1}\right\},
$$

then the central limit theorem holds, i.e.,

$$
\frac{S_{n}-n \mathbb{E}\left[Z_{n 1}\right]}{\sqrt{n \operatorname{Var}\left(Z_{n 1}\right)}} \stackrel{d}{\longrightarrow} \mathscr{N}(0,1), \quad n \rightarrow \infty ;
$$

(iii) otherwise, if (8) is not fulfilled, then for any constant $c>0$,

$$
\frac{S_{n}-n \mathbb{E}\left[Z_{n 1}\right]}{c n^{\left(1-\gamma_{2}\right) / \alpha}} \stackrel{d}{\longrightarrow} F_{\alpha, c}, \quad n \rightarrow \infty,
$$

see Theorem 2.1(iii) for notations.

\section{Proofs}

We first prove the statements related to the law of large numbers (Theorem 2.2 (i) and Theorem 2.3 (i)), then the central limit theorems (Theorem 2.1(i) and Theorem 2.3(ii)), and afterwards we show the convergence to stable non-Gaussian distributions (Theorem 2.1 (ii), (iii) and Theorem 2.3(iii)).

Proof of Theorem 2.2 (i) and Theorem 2.3 (i).

1. Denote

$$
\widetilde{S}_{n}=\frac{S_{n}}{\mathbb{E}\left[S_{n}\right]}-1=\frac{\sum_{k=1}^{n}\left(Z_{n k}-\mathbb{E}\left[Z_{n k}\right]\right)}{n \mathbb{E}\left[Z_{n 1}\right]}
$$

Our aim is to show that there exists a constant $r>1$ such that $\mathbb{E}\left|\widetilde{S}_{n}\right|^{r} \rightarrow 0$ as $n \rightarrow \infty$. This will imply that $\widetilde{S}_{n} \stackrel{p}{\longrightarrow} 0$, and therefore the result will follow.

Applying the Bahr-Esseen inequality for $r \in(1,2)$, see [10], we get that

$$
\begin{aligned}
\mathbb{E}\left[\left|\widetilde{S}_{n}\right|^{r}\right] & \leq C_{r} \frac{\sum_{k=1}^{n} \mathbb{E}\left[\left|Z_{n k}-\mathbb{E}\left[Z_{n k}\right]\right|^{r}\right]}{\left(n \mathbb{E}\left[Z_{n 1}\right]\right)^{r}} \\
& =C_{r} n^{1-r} \frac{\mathcal{M}_{n}(r)}{\left(\mathbb{E}\left[Z_{n 1}\right]\right)^{r}},
\end{aligned}
$$


where $C_{r}$ is some constant depending on $r$, and $\mathcal{M}_{n}(r)$ is the $r$-th absolute central moment of $Z_{n 1}$. The further analysis is consists in establishing the asymptotical behavior of the numerator and denominator of the fraction in (9).

2. Note that for any $n=1,2$, ..

$$
\mathbb{E}\left[Z_{n 1}\right]=\left(1-\varepsilon_{n}\right) \cdot \mu_{1}(1)+\varepsilon_{n} \cdot \mu_{2}(1),
$$

where $\mu_{1}(s)=\mathbb{E}\left[\left(X_{n 1}\right)^{s}\right]<\infty$ for any $s>0$, and

$$
\mu_{2}(s)=\mathbb{E}\left[\left(Y_{n 1}\right)^{s}\right]= \begin{cases}\frac{\alpha}{|s-\alpha|} M_{n}^{\max (s-\alpha, 0)}(1+o(1)), & \text { if } s \neq \alpha \\ \alpha \log \left(M_{n}\right)(1+o(1)), & \text { if } s=\alpha .\end{cases}
$$

Therefore,

$$
\mathbb{E}\left[Z_{n 1}\right]= \begin{cases}\left(\mu_{1}(1)+\frac{\alpha}{1-\alpha} \varepsilon_{n} M_{n}^{1-\alpha}\right)(1+o(1)), & \text { if } \alpha<1 \\ \left(\mu_{1}(1)+\alpha \varepsilon_{n} \log \left(M_{n}\right)\right)(1+o(1)), & \text { if } \alpha=1 \\ \mu_{1}(1)(1+o(1)), & \text { if } \alpha>1\end{cases}
$$

3. It holds for any $r>1$

$$
\begin{aligned}
& \mathcal{M}_{n}(r)= \mathbb{E}\left[\left|\left(1-B_{n 1}\right) X_{n 1}+B_{n 1} Y_{n 1}-\mathbb{E}\left[\left(1-B_{n 1}\right) X_{n 1}+B_{n 1} Y_{n 1}\right]\right|^{r}\right] \\
& \leq 4^{r-1}\left\{\mathbb{E}\left[\left(1-B_{n 1}\right)^{r}\right] \cdot \mathbb{E}\left[X_{n 1}^{r}\right]+\mathbb{E}\left[B_{n 1}^{r}\right] \cdot \mathbb{E}\left[Y_{n 1}^{r}\right]\right. \\
&\left.+\left(1-\varepsilon_{n}\right)^{r} \cdot\left(\mathbb{E} X_{n 1}\right)^{r}+\varepsilon_{n}^{r} \cdot\left(\mathbb{E} Y_{n 1}\right)^{r}\right\} \\
&= 4^{r-1}\left\{\left(1-\varepsilon_{n}\right) \mu_{1}(r)+\varepsilon_{n} \mu_{2}(r)+\left(1-\varepsilon_{n}\right)^{r}\left(\mu_{1}(1)\right)^{r}\right. \\
&\left.+\varepsilon_{n}^{r}\left(\mu_{2}(1)\right)^{r}\right\} .
\end{aligned}
$$

Denote $D_{r}=\mu_{1}(r)+\left(\mu_{1}(1)\right)^{r}$ and consider two cases:

(a) if $(\alpha<1, r>1)$ or $(1<\alpha \leq r)$, then it holds

$$
\mathcal{M}_{n}(r) \leq 4^{r-1}\left(D_{r}+\frac{\alpha}{r-\alpha} \varepsilon_{n} M_{n}^{r-\alpha}\right) \cdot(1+o(1)),
$$

where we use that $\varepsilon_{n} \mu_{2}(r) \gtrsim \varepsilon_{n}^{r}\left(\mu_{2}(1)\right)^{r}$ as $n \rightarrow \infty$;

(b) otherwise, if $(1<r<\alpha)$ or $(\alpha=1, r>1)$, then we have

$$
\mathcal{M}_{n}(r) \leq 4^{r-1} D_{r}(1+o(1)) .
$$


4. To conclude the proof, we substitute the upper estimate for $\mathcal{M}_{n}(r)$ and (10) into (9). If $\alpha<1$, then

$$
\mathbb{E}\left[\left|\widetilde{S}_{n}\right|^{r}\right] \leq \widetilde{C}_{r} \cdot n^{1-r} \frac{\left(c_{r}^{(1)}+\varepsilon_{n} M_{n}^{r-\alpha}\right) \cdot(1+o(1))}{\left(c_{r}^{(2)}+\varepsilon_{n} M_{n}^{1-\alpha}\right)^{r} \cdot(1+o(1))}
$$

with some constants $c_{r}^{(1)}, c_{r}^{(2)}$ (depending on $r$ ) and a bounded function $\widetilde{C}_{r}$. The asymptotic behaviour of the last fraction differs between the following two cases:

- $\varepsilon_{n} M_{n}^{1-\alpha} \rightarrow 0$, that is, $\gamma_{2}>(1-\alpha) \gamma_{1}$. Then there exists $r>1$ such that $\varepsilon_{n} M_{n}^{r-\alpha} \rightarrow 0$ - in fact, one can take $r=\left(\gamma_{2}-(1-\alpha) \gamma_{1}\right) \gamma_{1}^{-1}+1>1$. Under this choice of $r$, the r.h.s. of (11) tends to 0 , and therefore the law of large numbers holds for any $\left(\gamma_{1}, \gamma_{2}\right)$ s.t. $\gamma_{2}>(1-\alpha) \gamma_{1}$.

- $\varepsilon_{n} M_{n}^{1-\alpha} \rightarrow \infty$, that is, $\gamma_{2}<(1-\alpha) \gamma_{1}$. Then the right-hand side of (11) tends to 0 if and only if $M_{n}^{\alpha} /\left(n \varepsilon_{n}\right) \rightarrow 0$, that is, $\gamma_{2}<1-\alpha \gamma_{1}$. This case corresponds to the area $\gamma_{2}<\min \left\{(1-\alpha) \gamma_{1}, 1-\alpha \gamma_{1}\right\}$.

In other cases, $\alpha>1$ and $\alpha=1$, we can choose $r \in(1, \alpha)$ and get that $\mathbb{E}\left[\left|\widetilde{S}_{n}\right|^{r}\right] \lesssim n^{1-r}$, and therefore the law of large numbers holds with any positive $\gamma_{1}, \gamma_{2}$.

Proof of Theorem 2.1(i) and Theorem 2.3(ii) To prove these theorems, we check that the Lyapounov condition holds (see (27.16) from [2]): there exists $\delta>0$ such that

$$
\Omega_{n}:=\frac{\mathcal{M}_{n}(2+\delta)}{n^{\delta / 2}\left(\operatorname{Var}\left(Z_{n 1}\right)\right)^{1+\delta / 2}} \rightarrow 0, \quad \text { as } \quad n \rightarrow \infty .
$$

The variance of $Z_{n 1}$ has the following asymptotical behaviour:

$$
\begin{aligned}
\operatorname{Var}\left(Z_{n 1}\right) & =\left(1-\varepsilon_{n}\right) \mu_{1}(2)+\varepsilon_{n} \mu_{2}(2)-\left(\mathbb{E}\left[Z_{n 1}\right]\right)^{2} \\
& =\left(\left[\mu_{1}(2)-\left(\mu_{1}(1)\right)^{2}\right]+\frac{\alpha}{2-\alpha} \varepsilon_{n} M_{n}^{2-\alpha}\right) \cdot(1+o(1)), \quad n \rightarrow \infty,
\end{aligned}
$$

and the numerator of $\Omega_{n}$ was already considered in the proof of Theorem 2.2 (i). Therefore,

$$
\Omega_{n} \leq c_{1} \cdot \frac{\left(c_{2}+\varepsilon_{n} M_{n}^{2+\delta-\alpha}\right) \cdot(1+o(1))}{n^{\delta / 2}\left(c_{3}+\varepsilon_{n} M_{n}^{2-\alpha}\right)^{1+\delta / 2} \cdot(1+o(1))}, \quad n \rightarrow \infty
$$

with some positive constants $c_{1}, c_{2}, c_{3}$. The rest of the proof follows the same lines as Step 4 in the proof of Theorem 2.2(i), see above.

Proof of Theorem 2.1 (ii), (iii) and Theorem 2.3(iii). The proof is based on the following proposition, which is in fact a combination of Theorem 1.7.3 from [6], Theorem 3.2.2 from [7], and a number of theorems given in Chapter IV from [8]. 
Proposition 3.1. Consider an infinitesimal triangular array $\left\{Z_{n k}, k=1 . . k_{n}\right\}$, such that (1) is fulfilled. In what follows, we denote the distribution of $Z_{n k}$ by $\mu_{n k}$, and use the notation $S_{n}:=Z_{n 1}+\ldots+Z_{n k_{n}}$. The following statements hold.

1. If there exists a random variable $Y$ and a sequence of real numbers $a_{n}$ such that

$$
S_{n}-a_{n} \stackrel{d}{\longrightarrow} Y, \quad n \rightarrow \infty,
$$

then $Y$ has an infinitely divisible distribution; moreover, for any infinitely distribution $\mathbb{P}_{\text {inf }}$ there exists a triangular array $\left\{Z_{n k}, k=1 . . k_{n}\right\}$ such that $S_{n} \stackrel{d}{\longrightarrow} \mathbb{P}_{\text {inf }}$.

2. There exists a deterministic sequence $a_{n}$ such that sequence $S_{n}-a_{n}$ converges weakly to an infinitely divisible random variable $Y$ with characteristic exponent

$$
\psi(u)=\mathrm{i} u \mu-\frac{1}{2} u^{2} \sigma^{2}+\int_{\mathbb{R} /\{0\}}\left(e^{\mathrm{i} u x}-1-\mathrm{i} u x I I\{|x| \leq 1\}\right) \nu(d x),
$$

where $(\mu, \sigma, \nu)$ is a Lévy triplet, if and only if the following conditions are fulfilled:

(a) $\sum_{k=1}^{k_{n}} \mu_{n k}(A) \rightarrow \nu(A)$ for any $A=(-\infty, x)$ with $x<0$ and any $A=(x,+\infty)$ with $x>0$ such that $\nu(\partial A)=0$;

(b) moreover,

$$
\begin{aligned}
& \lim _{\tau \rightarrow 0} \limsup _{n \rightarrow \infty} \sum_{k=1}^{k_{n}}\left\{\int_{|x|<\tau} x^{2} \mu_{n k}(d x)-\left(\int_{|x|<\tau} x \mu_{n k}(d x)\right)^{2}\right\} \\
= & \lim _{\tau \rightarrow 0} \liminf _{n \rightarrow \infty} \sum_{k=1}^{k_{n}}\left\{\int_{|x|<\tau} x^{2} \mu_{n k}(d x)-\left(\int_{|x|<\tau} x \mu_{n k}(d x)\right)^{2}\right\}=\sigma^{2}
\end{aligned}
$$

If these conditions are satisfied, $a_{n}$ may be chosen according to the formula

$$
a_{n}=\sum_{k=1}^{k_{n}} \int_{|x|<1} x \mu_{n k}(d x)+o(1),
$$

provided $\nu\{x:|x|=1\}=0$.

3. There exists a deterministic sequence $a_{n}$ such that sequence $S_{n}-a_{n}$ converges weakly to a standard normal random variable $Y$ if and only if the following conditions are fulfilled:

(a) $\sum_{k=1}^{k_{n}} \mathbb{P}\left\{\left|Z_{n k}\right|>x\right\} \rightarrow 0$ as $n \rightarrow \infty$ for any $x>0$;

(b) $\lim _{n \rightarrow \infty} \sum_{k=1}^{k_{n}}\left\{\int_{|x|<\tau} x^{2} \mu_{n k}(d x)-\left(\int_{|x|<\tau} \mu_{n k}(d x)\right)^{2}\right\}=1$ for some $\tau>0$. 
If these conditions are satisfied, $a_{n}$ may be chosen according to (14).

Returning to our setup, we denote $F_{n k}(x)=\mathbb{P}\left\{Z_{n k} \leq \beta_{n} x\right\}$, and first note that for any $x \in\left(\beta_{n}^{-1}, \beta_{n}^{-1} M_{n}\right)$

$$
\begin{aligned}
& \sum_{k=1}^{k_{n}}\left(1-F_{n k}(x)\right)= \sum_{k=1}^{k_{n}}\left[1-\left(1-\varepsilon_{n}\right) F_{1}\left(\beta_{n} x\right)-\varepsilon_{n} \frac{F_{2}\left(\beta_{n} x\right)}{F_{2}\left(M_{n}\right)}\right] \\
&= n\left(1-\varepsilon_{n}\right)\left(1-F_{1}\left(\beta_{n} x\right)\right)+n \varepsilon_{n}\left(1-\frac{F_{2}\left(\beta_{n} x\right)}{F_{2}\left(M_{n}\right)}\right) \\
&= n\left(1-\varepsilon_{n}\right) e^{-\lambda \beta_{n} x}(1+o(1))+n \varepsilon_{n}\left(\beta_{n} x\right)^{-\alpha}(1+o(1)) \\
&-n \varepsilon_{n} M_{n}^{-\alpha}(1+o(1)) .
\end{aligned}
$$

Note that basically only 3 situations are possible.

1. $1-\alpha \gamma_{1}<\gamma_{2}<1$. In this case, under the choice $\beta_{n}=c_{1} n^{\left(1-\gamma_{2}\right) / \alpha}$ with any constant $c_{1}>0$ we get

$$
\sum_{k=1}^{k_{n}}\left(1-F_{n k}(x)\right) \rightarrow c_{1} x^{-\alpha}, \quad \forall x \in \mathbb{R}_{+},
$$

because $n e^{-\lambda \beta_{n}} \rightarrow 0, n \varepsilon_{n} \beta_{n}^{-\alpha} \rightarrow c_{1}$, and $n \varepsilon_{n} M_{n}^{\alpha} \rightarrow 0$. Moreover, the condition (1) is fulfilled - in fact, for any $\delta>0$, it holds

$$
\sup _{k=1 . . k_{n}} \mathbb{P}\left\{\left|Z_{n k}\right|>\delta\right\}=k_{n}^{-1} \sum_{k=1}^{k_{n}}\left(1-F_{n k}(\delta)\right) \rightarrow 0 .
$$

Next, with any $s \geq 1$,

$$
\begin{aligned}
\int_{|x|<\tau} x^{s} \widetilde{\mathbb{P}}_{1}(d x) & =\beta_{n}^{-s} \cdot \mathbb{E}\left[X_{n 1}^{s}\right](1+o(1)), \\
\int_{|x|<\tau} x^{s} \widetilde{\mathbb{P}}_{2}(d x) & = \begin{cases}\beta_{n}^{-\min (\alpha, s)} \tau^{\max (s-\alpha, 0)} \frac{\alpha}{|s-\alpha|}(1+o(1)), & \text { if } \alpha \neq s ; \\
\alpha \beta_{n}^{-s} \log \left(\beta_{n}\right)(1+o(1)), & \text { if } \alpha=s .\end{cases}
\end{aligned}
$$

where $\widetilde{\mathbb{P}}_{1}, \widetilde{\mathbb{P}}_{2}$ are the probability distributions of $X_{n 1} / \beta_{n}$ and $Y_{n 1} / \beta_{n}$ resp. Therefore, if $\alpha<1$, the condition (13) reads as

$$
\begin{aligned}
G_{n} & :=\sum_{k=1}^{k_{n}}\left\{\int_{|x|<\tau} x^{2} \mu_{n k}(d x)-\left(\int_{|x|<\tau} x \mu_{n k}(d x)\right)^{2}\right\} \\
& =n\left\{\beta_{n}^{-2} \mathbb{E}\left[X_{n 1}^{2}\right]+C_{1} \varepsilon_{n} \beta_{n}^{-\alpha} \tau^{2-\alpha}-\left(\beta_{n}^{-1} \mathbb{E}\left[X_{n 1}\right]+R_{n}\right)^{2}\right\}(1+o(1))
\end{aligned}
$$

where

$$
R_{n}= \begin{cases}C_{2} \varepsilon_{n} \beta_{n}^{-\min (1, \alpha)} \tau^{1-\alpha}, & \text { if } \alpha \neq 1, \\ C_{3} \varepsilon_{n} \beta_{n}^{-1} \log \left(\beta_{n}\right), & \text { if } \alpha=1\end{cases}
$$


and $C_{1}, C_{2}, C_{3}>0$. We conclude that if $n \beta_{n}^{-2} \rightarrow 0$ (that is, $\gamma_{2}<1-\alpha / 2$ ), then $\lim _{\tau \rightarrow 0} \lim _{n \rightarrow \infty}\left[G_{n}\right]=0$; otherwise the last limit is infinite. At the same time, (14) yields for $\alpha \neq 1$,

$$
a_{n}=n\left[\frac{1-\varepsilon_{n}}{\beta_{n}} \mathbb{E}\left[X_{n 1}\right]+\frac{\varepsilon_{n}}{\beta_{n}^{\min (1, \alpha)}} \frac{\alpha}{|1-\alpha|}\right]+o(1) .
$$

For instance, if $\alpha<1$, then

$$
a_{n}=\frac{n \mathbb{E}\left[X_{n 1}\right]}{\beta_{n}}+\frac{\alpha}{1-\alpha}+o(1),
$$

where the first summand in the r.h.s. is of the order $n^{1-\left(1-\gamma_{2}\right) / \alpha}$. Therefore, the choice of $a_{n}$ differs in the cases $\gamma_{2} \in(1-\alpha, 1-\alpha / 2), \gamma_{2}=1-\alpha$, and $\gamma_{2} \in(0,1-\alpha)$, and this observation leads to different choices of $\kappa_{n}=a_{n} \beta_{n}$, see (5). Finally, in the case $\alpha=1$,

$$
a_{n}=n\left[\frac{1-\varepsilon_{n}}{\beta_{n}} \mathbb{E}\left[X_{n 1}\right]+\frac{\varepsilon_{n} \alpha}{\beta_{n}} \log \left(\beta_{n}\right)\right]+o(1)=\frac{n \mathbb{E}\left[Z_{n 1}\right]}{\beta_{n}}+o(1),
$$

where we use (10).

2. $\gamma_{2} \in\left(1-\alpha / 2,(2-\alpha) \gamma_{1}\right)$. In this case, we take $\beta_{n}=\sqrt{n \operatorname{Var}\left(X_{n 1}\right)}$. Under this choice, the conditions (a) and (b) from Part 3 of Proposition 3.1 hold. The choice $a_{n}=n \mathbb{E}\left[X_{n 1}\right] / \beta_{n}$ follows from (15).

3. $\gamma_{2}<1-\alpha \gamma_{1}$. It is easy to see that the infinite smallness condition (1) is not fulfilled. Note that this case was considered separately in Theorem 2.1 (i).

Proof of Theorem 2.2 (ii) and (iii). The proof directly follows from the application of the well-known Slutsky theorem. For instance, Theorem 2.1(ii) yields that

$$
\frac{S_{n}-n \mathbb{E}\left[X_{n 1}\right]}{n \mathbb{E}\left[X_{n 1}\right]}=\frac{S_{n}-n \mathbb{E}\left[X_{n 1}\right]}{\sqrt{n \operatorname{Var}\left(X_{n 1}\right)}} \cdot \frac{\sqrt{\operatorname{Var}\left(X_{n 1}\right)}}{\sqrt{n} \mathbb{E}\left[X_{n 1}\right]} \stackrel{p}{\longrightarrow} 0,
$$

since the first multiplier tends in distribution to the standard normal law, and the second tends to 0 .

\section{Acknowledgment}

The author is grateful to Prof. Stanislav Molchanov (UNC Charlotte, USA, and Higher School of Economics, Moscow, Russia) for the supervision of this research.

\section{References}

[1] Ben Arous, G. and Bogachev, L. and Molchanov, S. Limit theorems for sums of random exponentials. Probability theory and related fields, 132(4):579-612, 2005. 
[2] Billingsley, P. Probability and measure. Wiley and Sons, 3rd edition, 1995.

[3] Bovier, A., Kurkova, I., and Löwe, M. Fluctuations of the free energy in the REM and the p-spin SK models. The Annals of Probability, 30(2):605-651, 2002.

[4] Embrechts, P., Klüppelberg, C., and Mikosch, T. Modelling extremal events for insurance and finance. Springer, 1997.

[5] Grabchak, M and Molchanov, S. Limit theorems and phase transitions for two models of summation of independent identically distributed random variables with a parameter. Theory Prob. Appl., 59(2):222-243, 2014.

[6] Ibragimov, I. and Linnik, Yu. Independent and Stationary Sequences of Random Variables. Walters-Noordoff, 1971.

[7] Meerschaert, M. and Scheffler, H.-P. Limit distributions for sums of independent random vectors: Heavy tails in theory and practice, volume 321. John Wiley \& Sons, 2001.

[8] Petrov, V. Sums of independent random variables, volume 82. Springer Science \& Business Media, 2012.

[9] Rolski, T., Schmidli, H., Schmidt, V., Teugels, J. Stochastic processes for insurance and finance. Wiley series in probability and statistics. J. Wiley, 1999.

[10] von Bahr, B. and Esseen, C.-G. Inequalities for the $r$ th absolute moment of a sum of random variables, $1 \leq r \leq 2$. The Annals of Mathematical Statistics, 36(1):299-303, 1965.

[11] Whitmeyer, J., and Yang, H. Baseline models of spatial population dynamics. The Journal of Mathematical Sociology, 40(2):123-135, 2016. 